\title{
Estudo morfológico e morfométrico do Forame Magno e dos Côndilos Occipitais em crânios secos do Mato Grosso do Sul
}

Morphological and morphometric study of Foramen Magno and Occipital Condits in dry skulls of Mato Grosso do Sul

Estudio morfológico y morfométrico de Foramen Magno y Condits Occipitales en cráneos secos de Mato Grosso do Sul

Gabriela Batista Lima Mateus

ORCID: https://orcid.org/0000-0001-8017-4462 Universidade Federal de Mato Grosso do Sul, Brasil E-mail: gabi.gm6@gmail.com

Kelly Regina Torres-da-Silva

ORCID: https://orcid.org/0000-0003-4108-3191

Faculdades Integradas de Três Lagoas, Brasil E-mail: kellytorresdasilva1@gmail.com

Aline Rafaela da Silva Rodrigues Machado ORCID: https://orcid.org/0000-0002-2977-075X Universidade Federal do Mato Grosso do Sul, Brasil E-mail: aline.r.machado@ufms.br

Pablo Felipe de Almeida

ORCID: https://orcid.org/0000-0002-9135-6816 Universidade Federal do Mato Grosso do Sul, Brasil E-mail: pablo.almeida@ufms.br

André Valério Silva

ORCID: https://orcid.org/0000-0003-0309-5394 Universidade Federal do Mato Grosso do Sul, Brasil E-mail: andre.valerio@ufms.br

\begin{abstract}
Resumo
A análise morfométrica do forame magno (FM) e dos côndilos occipitais (CO) é imprescindível para compreensão clínica de determinadas afecções, para alicerçar estudos anatômicos e a sua descrição é de extrema valia na anatomia forense. Analisou-se aspectos morfológicos e morfométricos do FM e dos CO em 107 crânios secos. Com paquímetro coletou-se os diâmetros anteroposterior (DA) e transverso (DT) de cada FM e anteroposterior (AP), médio-lateral (ML) dos CO. Os resultados foram descritos em médias, para o FM: DA em crânios masculinos e femininos foram: $36,11 \mathrm{~mm}$ $\pm 2,55 ; 35,26 \mathrm{~mm} \pm 2,91$; DT: $30,24 \mathrm{~mm} \pm 2,22,29,8 \mathrm{~mm}$, respectivamente. Os CO em crânios masculinos: AP lado direito 19,81 mm, lado esquerdo 20,43 mm; ML do lado direito 12,79mm, lado esquerdo 12,64mm. Os CO em crânios femininos: AP do lado direito 21,00 mm, lado esquerdo 21,19 mm; distância ML lado direito 12,67 mm, lado esquerdo $13,16 \mathrm{~mm}$. Foi identificado a seguinte morfologia do FM: oval (59\%), ovo (8\%), losango (9\%), circular (10\%), hexagonal (5\%) ou irregular (7\%); a forma pentagonal $(0,9 \%)$ somente em crânios femininos. Percebe-se que os resultados do FM e dos CO estão de acordo com a literatura (FM: predomina formato oval, com medida ML significamente maior em crânios masculinos). Nota-se que estudos morfométricos contribuem para a anatomia forense e a compreensão clínica e cirúrgica das afecções que englobam o FM, portanto estudos nesse âmbito são essenciais. Palavras-chave: Crânio; Forame magno; Anatomia.
\end{abstract}

\begin{abstract}
The morphometric analysis of the foramen magnum (FM) and occipital condyles (OC) is essential for the clinical understanding of certain conditions, to support anatomical studies, and their description is extremely valuable in forensic anatomy. Morphological and morphometric aspects of FM and CO were analyzed in 107 dry skulls. With a caliper, the anteroposterior (AD) and transverse (DT) diameters of each FM and anteroposterior (AP), mediolateral (ML) of the OCs were collected. The results are described as means for FM: DA in male and female skulls were: $36.11 \mathrm{~mm} \pm 2.55$; $35.26 \mathrm{~mm} \pm 2.91$; DT: $30.24 \mathrm{~mm} \pm 2.22,29.8 \mathrm{~mm}$, respectively. OC in male skulls: AP right side $19.81 \mathrm{~mm}$, left side $20.43 \mathrm{~mm}$; ML on the right side $12.79 \mathrm{~mm}$, left side $12.64 \mathrm{~mm}$. OC in female skulls: AP right side $21.00 \mathrm{~mm}$, left side $21.19 \mathrm{~mm}$; distance ML right side $12.67 \mathrm{~mm}$, left side $13.16 \mathrm{~mm}$. The following FM morphology was identified: oval (59\%), egg-shaped (8\%), diamond (9\%), circular (10\%), hexagonal $(5 \%)$ or irregular $(7 \%)$; the pentagonal shape $(0.9 \%)$ only in female skulls. It can be seen that the FM and CO results are in agreement with the literature (FM: oval shape
\end{abstract}


predominates, with a significantly larger ML measurement in male skulls). It is noted that morphometric studies boost forensic anatomy and the clinical and surgical understanding of the conditions that encompass FM, therefore studies in this scope are essential.

Keywords: Skull; Foramen magnum; Anatomy.

\section{Resumen}

El análisis morfométrico del foramen magnum (FM) y los cóndilos occipitales (OC) es fundamental para la comprensión clínica de determinadas afecciones, para respaldar los estudios anatómicos, y su descripción es sumamente valiosa en anatomía forense. Se analizaron aspectos morfológicos y morfométricos de FM y CO en 107 cráneos secos. Con un calibre se recogieron los diámetros anteroposteriores (AD) y transversal (DT) de cada FM y anteroposterior (AP), mediolateral (ML) de los OC. Los resultados se describen como medias para FM: DA en cráneos masculinos y femeninos: 36,11 $\mathrm{mm} \pm 2,55 ; 35,26 \mathrm{~mm} \pm 2,91$; DT: 30,24 $\mathrm{mm} \pm 2,22,29,8 \mathrm{~mm}$, respectivamente. OC en cráneos masculinos: AP lado derecho 19,81 mm, lado izquierdo 20,43 mm; ML en el lado derecho 12,79 mm, lado izquierdo 12,64 mm. OC en cráneos femeninos: AP lado derecho 21,00 mm, lado izquierdo 21,19 mm; distancia ML lado derecho 12,67 mm, lado izquierdo 13,16 mm. Se identificó la siguiente morfología de la FM: ovalada (59\%), ovalada (8\%), romboidal (9\%), circular (10\%), hexagonal (5\%) o irregular (7\%); la forma pentagonal $(0,9 \%)$ solo en cráneos femeninos. Se observa que los resultados de FM y CO están de acuerdo con la literatura (FM: predomina la forma ovalada, con una medición de ML significativamente mayor en cráneos masculinos). Se observa que los estudios morfométricos potencian la anatomía forense y la comprensión clínica y quirúrgica de las condiciones que engloban la FM, por lo que los estudios en este ámbito son fundamentales.

Palabras clave: Cráneo; Foramen magnum; Anatomía.

\section{Introdução}

O forame magno (FM) situa-se em uma posição anteromediana e conduz para a fossa posterior do crânio. É oval e mais largo na parte de trás, sendo que seu maior diâmetro é o anteroposterior. Ele contém a extremidade inferior do bulbo, meninges, artérias vertebrais, nervo acessório (NC XI), o ligamento do ápice do dente (áxis) e a membrana tectória, os quais passam por ali para anexarem-se ao occipício internamente. Anteriormente, a margem do forame magno é ligeiramente sobreposta pelos côndilos occipitais, que se projetam para baixo para articular com as faces articulares superiores sobre as massas laterais do atlas. Cada côndilo occipital tem delineamento oval e é orientado de maneira que sua extremidade anterior se situa mais perto da linha mediana do que a sua extremidade posterior. Em sua face anteroposterior, é acentuadamente convexo, mas menos convexo transversalmente, e sua face medial é áspera devido aos anexos ligamentares. A parte escamosa do osso occipital exibe a protuberância occipital externa, linhas nucais supremas, superior e inferior e crista occipital externa, sendo que todos se situam na linha mediana, posteriores ao forame magno. A região é áspera devido à fixação de músculos (Tortora, 2013; Grays, 2011), estas estruturas podem ser observadas na Figura 1.

Figura 1: Fotografia da base de um dos crânios utilizados no estudo. Forame Magno (FM) e estruturas adjacentes: côndilo occipital (CO), basion (B) e opisthion (OP). Diâmetro transverso (DT) e diâmetro anteroposterior (DA) do FM e AP e ML do CO.

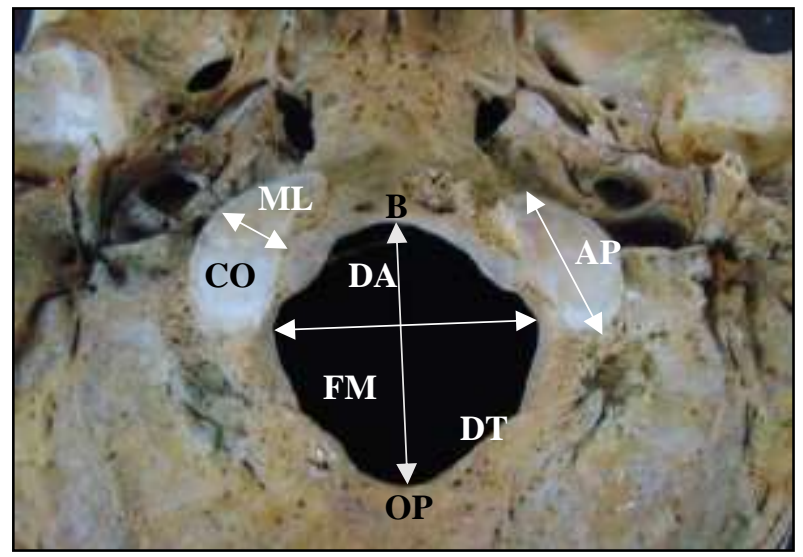

Fonte: Autores. 
O FM é uma porção anatômica de grande importância, pois é protegido (na maioria das situações) de lesões relacionadas ao fogo, explosões e mutilações. Isso se deve ao fato de fortes músculos e ligamentos existirem neste local. Ademais, o crânio parece ser pouco acometido por condições como a nutrição e é mais influenciado por aspectos genéticos. O FM se desenvolve a partir de esclerótomos dos 4 primeiros somitos que ocasionalmente se fundem para formar o osso occipital e o elemento posterior do forame magno. O quarto esclerótomo (proatlas) possui 3 porções: o hipocentro, o centro e o arco neural, este último se divide em porções ventro-rostrais e dorsal-caudais. O componente ventro-rostral origina os côndilos occipitais e a margem anterior do FM. Desse modo, anomalias morfológicas e malformações neste processo são capazes de formar diferentes tipos morfológicos de FM (Pires et al., 2016).

Com respeito a sua morfologia, a análise da forma e do tamanho do FM é imprescindível para determinar alterações patológicas desencadeadas por doenças como: acondroplasia, invaginação basilar, distrofia torácica, meningioma do forame magno e síndrome de Arnold-Chiari (Figueiredo et al., 2021). Essas condições podem resultar em compressão das estruturas que atravessam o FM e gerar sintomas como complicações respiratórias, disfunções de nervos cranianos mais baixos, paresia dos membros inferiores, hipo ou hipertonia, hiperreflexia e atraso geral durante o desenvolvimento motor (Pires et al., 2016; Leonariza et al., 2017).

Ainda sobre aspectos clínicos, há algumas síndromes que são capazes de promover alterações morfológicas do FM como as craniossinostoses, que são desencadeadas pela precoce fusão das suturas cranianas. Essas sincondroses fundidas prematuramente interropem o crescimento do FM, com tamanho reduzido não acomoda o tronco cerebral da forma adequada e pode não haver espaço suficiente para a passagem no líquido cefalorraquidiano entre a cavidade craniana e o saco dural, o que pode contribuir para o desenvolvimento de um quadro de ventriculomegalia e uma elevação da pressão intracraniana (RIJKEN et al., 2015).

Da mesma forma, a acondroplasia, que é uma condição congênita e gera desordens neurológicas em $20 \%$ a $47 \%$ dos seus portadores, apresenta o FM diminuto ao nascimento e não demostra um padrão de crescimento normal nos primeiros 18 meses de vida, de modo que pode ocasionar estenose precoce da junção crânio-cervical com provável compressão da medula espinhal cervical alta e consequente comprometimento neurológico (Fornarino et al., 2016).

É imprescindível ressaltar que esse estudo busca compatilhar com o âmbito anâtomico e, consequentemente, com a clínica médica, um conjunto de dados e análises acerca da morfologia do FM em crânios secos do Mato Grosso do Sul. Desse modo, ao amplificar a gama de conhecimentos, espera-se que patologias que envolvam tal forame sejam mais desmistificadas e melhor compreendidas. Assim, almeja-se que esse trabalho corrobore com a edificação de futuras pesquisas e projetos que envolvam tal temática, que ao fim poderão proporcionar benefícios ao corpo social acometido por afecções e técnicas de correção cirúrgica relacionadas com o FM.

\section{Metodologia}

Para este estudo, foram selecionados 107 crânios humanos do acervo do Laboratório de Anatomia da Universidade Federal do Mato Grosso do Sul - Campus de Três lagoas - MS. Deste total 55 crânios correspondiam ao gênero masculino e 52 ao gênero feminino.

Os critérios de inclusão foram boa condição geral da fossa posterior do crânio e do FM e dos CO. Ao mesmo tempo que os critérios de exclusão foram crânios com FM ou CO danificados ou quebrados.

A classificação dos crânios, de acordo com o gênero, foi realizada com base nas características anatômicas prevalentes seguindo padrões antropométricos comprovados na literatura para a sexagem cranial (Vanrell, 2009). Esta classificação foi realizada por três técnicos devidamente treinados e capacitados. Os acidentes anatômicos utilizados como parâmetros para a 
sexagem cranial foram fronte, glabela, apófises mastoides, protuberância occipital externa e as coroas dos molares superiores e o côndilo occipital, conforme trabalhos prévios já publicados (De lima godas et al., 2020).

Com auxílio de um paquímetro analógico 6" da marca Zaas Precision foram coletados dados morfométricos dos diâmetros anteroposterior (DA) e transverso (DT) de cada FM e de cada CO, assim como foram visualizados seus aspectos morfológicos. O DA do FM compreende a distância entre o basion e o opisthion; e o DT é a distância entre os pontos de máxima curvatura das margens laterais do FM. O diâmetro anteroposterior (AP) do CO remete ao comprimento máximo de sua extremidade anterior para a posterior; e o diâmetro médio-lateral (ML) do CO é a distância máxima entre as bordas laterais.

Cada diâmetro foi medido duas vezes, no intuito de minimizar quaisquer erros. A área do FM foi calculada utilizando a fórmula de Radinsky's (A = 1/4 x pi x AP x ML), e a morfologia de cada FM foi classificada em oval, redondo, tetragonal, forma de ovo, pentagonal ou hexagonal de acordo com outros trabalhos (Pires et al., 2016).

\section{Resultados}

Os resultados estão descritos em médias. Em relação a morfometria do FM, em crânios masculinos obteve-se a média DA de 36,11 mm com desvio padrão de $\pm 2,55$ e a média DT de 30,24 mm com desvio padrão de $\pm 2,22$. Em crânios femininos verificou-se que a média DA foi de 35,26 mm com desvio padrão de $\pm 2,91$ e que a média DT foi de 29,8 $\mathrm{mm}$ com desvio padrão de $\pm 2,9$. Esses dados foram agrupados no Gráfico 1 .

Gráfico 1: Gráfico boxplot (range mínimo, máximo com mediana) diâmetro transverso (DT) e diâmetro anteroposterior (DA) do FM, em ambos os grupos.

\section{DA vs DT vs sexo}

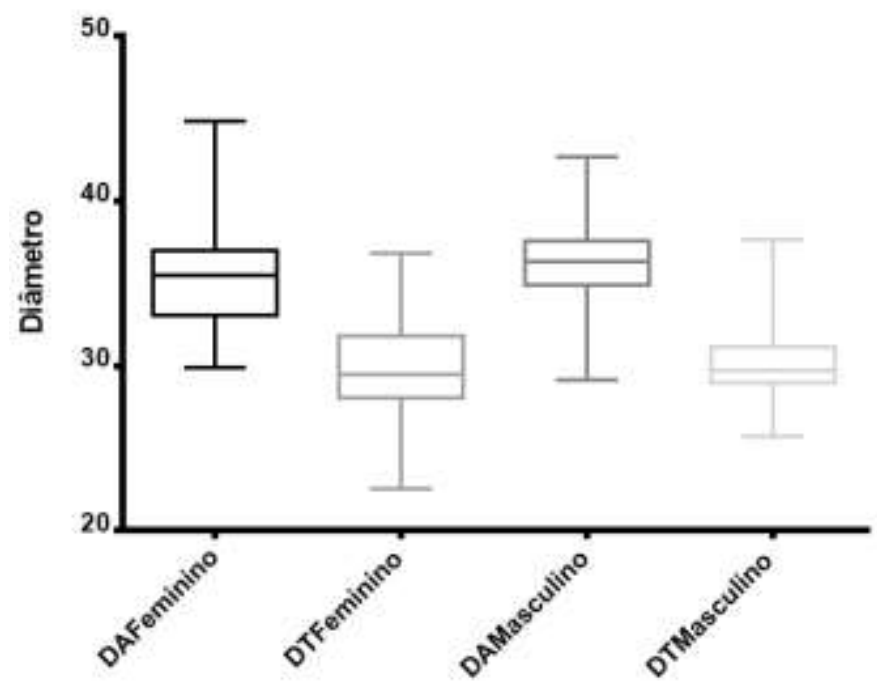

Fonte: Autores.

Quando aplicado o Teste t, este não demonstrou diferenças relevantes entre as amostras, no que corresponde ao DA. Porém, em relação ao DT foi detectada uma diferença significativa entre os grupos ( $\mathrm{p}=0,03)$, sendo que a média DT em crânios masculinos é maior quando comparada à média de crânios femininos. A área média do FM em crânios masculinos foi 860,00 (desvio padrão $\pm 105,33$ ) e femininos $827,73 \mathrm{~mm}$ (desvio padrão $\pm 126,76$ ), quando aplicado o Teste t, este não demonstrou diferença entre as médias das áreas ( $\mathrm{t}=1.98)$. 
Research, Society and Development, v. 10, n. 12, e560101220742, 2021

(CC BY 4.0) | ISSN 2525-3409 | DOI: http://dx.doi.org/10.33448/rsd-v10i12.20742

No quesito morfologia do FM, foi identificado que a forma oval predomina nas amostras (59\%), porém outras formas também foram detectadas, como a circular (10\%), losango (9\%), ovo (8\%), forma irregular (7\%), hexagonal (5\%) e a pentagonal $(0,9 \%)$, com a ressalva de que a última foi encontrada apenas em crânios femininos, conforme demonstrado na Figura 2.

Figura 2: Morfologias do FM identificadas na pesquisa, figuras de A - F: FM com forma tipo ovo figura A, circular B, hexagonal C, losango D, oval E, pentagonal F. CO = côndilos occipitais.

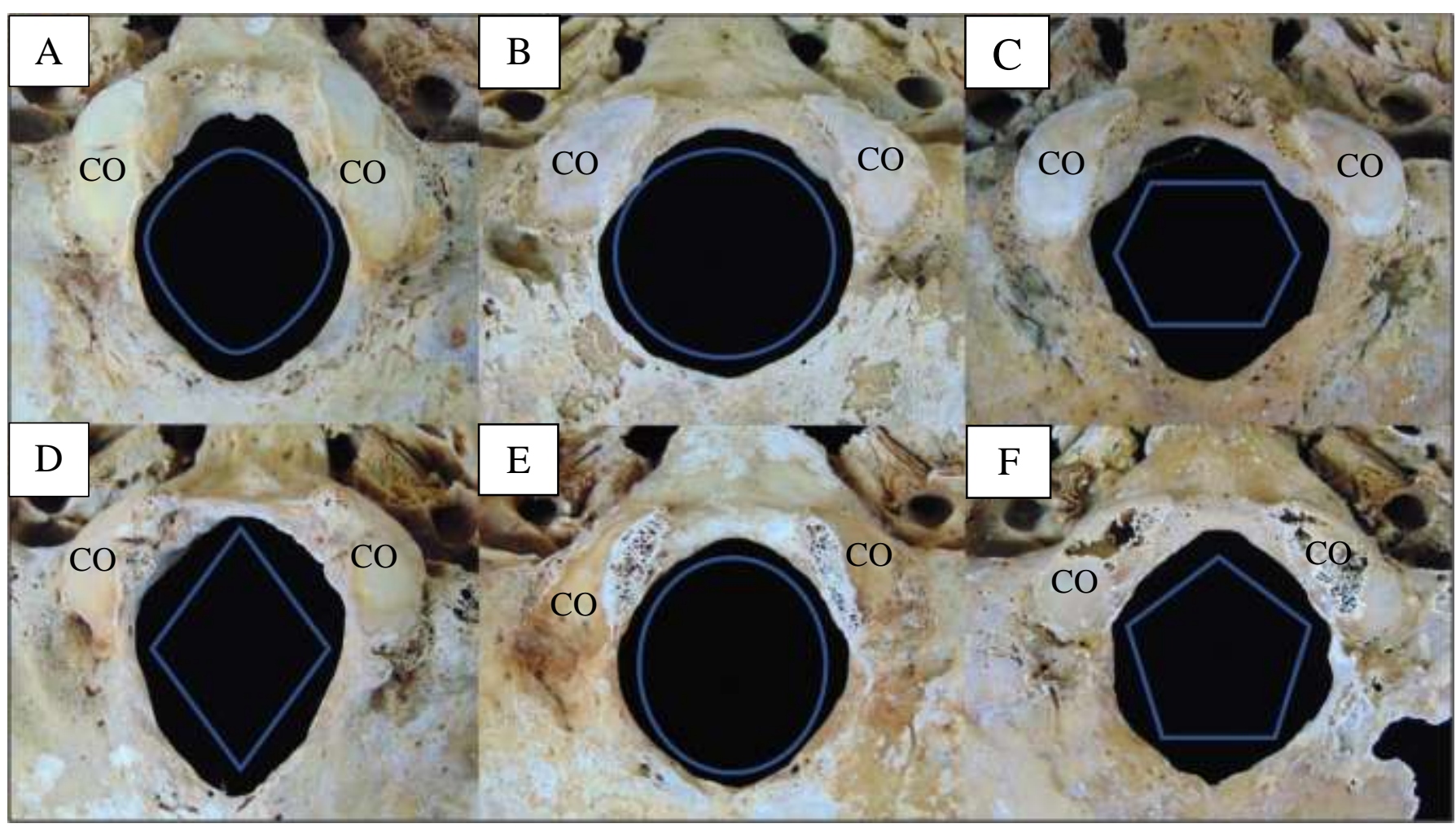

Fonte: Autores.

Através do Teste Qui-quadrado não foram observadas diferenças significativas ( $\mathrm{p}=0,8)$ entre ambos os sexos, no que diz respeito a morfologia do FM, Gráfico 2. 
Gráfico 2: Esse gráfico representa os aspectos morfológicos do FM detectados para ambas as amostras pesquisadas.

\section{Formato do Forame Magno}

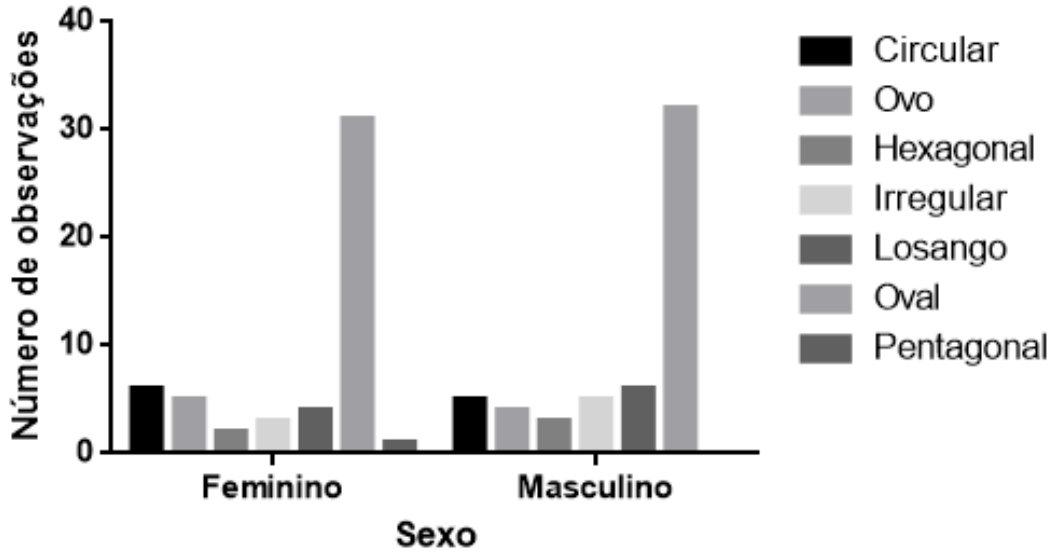

Fonte: Autores.

Ao se tratar dos CO, verificou-se que em crânios masculinos, a média AP do lado direito foi 19,81 mm e do lado esquerdo foi 20,43 mm. Enquanto a média ML do lado direito foi 12,79 mm e do lado esquerdo foi 12,64 mm. Em crânios femininos, a média AP do lado direito foi 21,00 $\mathrm{mm}$ e do lado esquerdo foi 21,19 $\mathrm{mm}$. E ao se tratar das médias ML, do lado direito foi $12,67 \mathrm{~mm}$ e do lado esquerdo foi $13,16 \mathrm{~mm}$.

A análise estatística nos mostra que do lado direito em relação à medida AP entre os sexos, assumindo igualdade de variância, não foi observada diferença significativa entre os grupos ( $\mathrm{p}=0,1509)$, da mesma forma a medida ML também não tem diferença entre os sexos ( $\mathrm{p}=0,3921)$. Com respeito a análise do lado esquerdo a medida AP entre os sexos, assumindo igualdade de variância, não foi observada diferença significativa entre os grupos ( $\mathrm{p}=0,7391)$, porém a medida ML tem diferença entre os sexos ( $<<0,001)$, ou seja, temos evidências de que a média da medida ML do lado esquerdo no grupo feminino é maior em relação à média da medida ML do lado esquerdo no grupo masculino na população estudada, de acordo com o Gráfico 3.

Gráfico 3: Distribuição da medida ML do lado esquerdo no sexo feminino (A) e masculino (B).
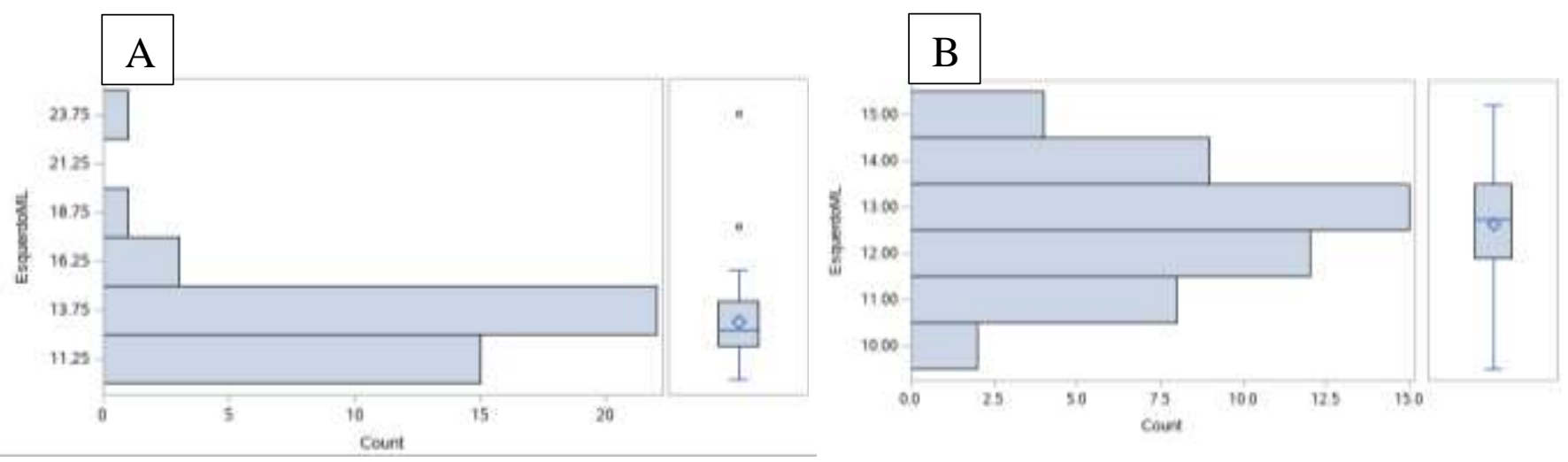

Fonte: Autores.

\section{Discussão}

Considerando o forame magno (FM) uma estrutura importante para o estudo da base do crânio devido as relações entre as estruturas neurovasculares que o atravessam, seu variado aspecto e tamanho, assim como a relevância de sua abordagem 
microcirúrgica em certas condições clínicas, compreender e estudar tal estrutura chama atenção de muitos neurocirurgiões, anatomistas e outros especialistas da área.

Em nosso trabalho, encontramos alguns aspectos morfométricos interessantes e constatamos que na literatura mundial existem resultados muito semelhantes (Quadro 1), por exemplo, nossas médias se assemelham a um estudo realizado na Nigéria que revelou comprimento e largura médios do FM de 36,11mm $\pm 2,60$ e 29,56mm \pm 2,60 respectivamente, no qual foram examinados 120 crânios secos adultos, não tendo o sexo como uma das variáveis. O intuito anatômico desse trabalho desempenhado em solo africano foi demonstrar variações antropométricas (Osunwoke et al., 2012), proporcionando alicerces científicos à antropologia e à anatomia forense.

Quadro 1: Dados do FM do atual estudo e dos demais estudos citados no trabalho.

\begin{tabular}{|c|c|c|c|c|}
\hline AUTOR & $\begin{array}{c}\text { NACIONALIDA } \\
\text { DE }\end{array}$ & $\begin{array}{c}\text { ANO DE } \\
\text { PUBLICAÇ } \\
\text { ÃO }\end{array}$ & METODOLOGIA & RESULTADOS \\
\hline $\begin{array}{l}\text { Presente } \\
\text { estudo }\end{array}$ & Brasil & 2021 & $\begin{array}{l}\text { Paquímetro analógico; Análises } \\
\text { estatísticas: Teste Qui-quadrado, } \\
\text { Teste T. }\end{array}$ & $\begin{array}{l}\text { FM do sexo masculino }=36,11 \mathrm{~mm} \pm 2,55 ; \mathrm{FM} \text { do } \\
\text { sexo masculino }=30,24 \mathrm{~mm} \pm 2,22 ; \mathrm{FM} \text { do sexo } \\
\text { feminino }=35,26 \mathrm{~mm} \pm 2,91 ; \mathrm{FM} \text { do sexo } \\
\text { feminino }=29,8 \mathrm{~mm} \pm 2,9 . \text { Forma mais comum do } \\
\mathrm{FM}=\text { forma oval, em ambos os sexos. }\end{array}$ \\
\hline Biswas et al. & Bengali - Índia & 2015 & $\begin{array}{l}\text { Paquímetro digital Análises } \\
\text { estatísticas: SPSS version } 19.0 \\
\text { computer software. }\end{array}$ & $\begin{array}{l}\text { FM do sexo masculino }=34,02 \mathrm{~mm} ; \mathrm{FM} \text { do sexo } \\
\text { feminino }=33,03 \mathrm{~mm} \text {; FM do sexo masculino }= \\
28,1 \mathrm{~mm} \text {; FM do sexo feminino }=27,46 \mathrm{~mm} .\end{array}$ \\
\hline $\begin{array}{l}\text { Osunwo et } \\
\text { al. }\end{array}$ & Nigéria & 2012 & $\begin{array}{l}\text { Paquímetro digital Vernier. } \\
\text { Análises estatísticas: Teste } \mathrm{Z} \text {. }\end{array}$ & $\mathrm{FM}=36,11 \mathrm{~mm} \pm \mathbf{2 , 6 0} ; \mathrm{FM}=29,56 \mathrm{~mm} \pm \mathbf{2 , 6 0}$ \\
\hline Pires et al. & Brasil & 2016 & $\begin{array}{l}\text { Paquímetro digital Análises } \\
\text { estatísticas: Teste T. }\end{array}$ & $\begin{array}{l}\mathrm{FM}=34,23 \mathrm{~mm} \pm 2,54 ; \mathrm{ML} \text { do } \mathrm{FM}=28,62 \mathrm{~mm} \pm \\
2,83 ; \text { Forma mais comum do } \mathrm{FM}=\text { forma oval. }\end{array}$ \\
\hline $\begin{array}{l}\text { Lopez-capp } \\
\text { et al. }\end{array}$ & Brasil & 2018 & $\begin{array}{l}\text { Paquímetro digital Análises } \\
\text { estatísticas: Teste T. }\end{array}$ & $\begin{array}{l}\text { FM do sexo masculino }=32,26 \mathrm{~mm} \pm \mathbf{3}, 26 ; \text { ML do } \\
\text { FM do sexo masculino }=33,7 \mathrm{~mm} \pm 3,62 ; \text { AP do } \\
\text { FM do sexo feminino }=30,79 \mathrm{~mm} \pm \mathbf{3}, 3 ; \text { ML do } \\
\text { FM do sexo feminino }=32,09 \mathrm{~mm} \pm 3,04 .\end{array}$ \\
\hline Cirpan et al. & Turquia & 2016 & $\begin{array}{l}\text { Paquímetro } \\
\text { estatísticas: SPSS 16.0 }\end{array}$ & $\begin{array}{l}\text { AP do } F M=34,38 \mathrm{~mm} \pm 2,38 ; \mathrm{ML} \text { do } \mathrm{FM}= \\
28,95 \mathrm{~mm} \pm 2,19 ; \mathrm{FM}=\text { forma redonda }\end{array}$ \\
\hline Samara et al. & Jordânia & 2017 & $\begin{array}{l}\text { Imagens de } \quad \text { tomografia } \\
\text { computadorizada e uma régua } \\
\text { integrada Análises } \\
\text { Testatísticas: } \\
\text { T. }\end{array}$ & $\begin{array}{l}\text { AP do FM do sexo masculino }=35,8 \mathrm{~mm} \pm 3,0 ; \\
\text { ML do FM do sexo masculino }=30,3 \mathrm{~mm} \pm 2,3 ; \\
\text { AP do FM do sexo feminino }=34,4 \mathrm{~mm} \pm 3,1 ; \mathrm{ML} \\
\text { do FM do sexo feminino }=28,3 \mathrm{~mm} \pm 2,3 ; \mathrm{FM}= \\
\text { forma irregular, em ambos os sexos. }\end{array}$ \\
\hline Luzzi et al. & Itália & 2019 & $\begin{array}{lr}\text { paquímetro e } & \text { tomografias } \\
\text { computadorizadas; } & \text { Análises } \\
\text { estatísticas: Teste T. } & \end{array}$ & AP do FM: $35,3 \mathrm{~mm} \pm 4 ;$ ML do FM: $30,4 \mathrm{~mm} \pm 3$ \\
\hline $\begin{array}{l}\text { Edward et } \\
\text { al. }\end{array}$ & Suíça & 2013 & $\begin{array}{l}\text { Tomografias } \\
\text { computadorizadas; } \quad \text { Análises } \\
\text { estatísticas: Teste T. }\end{array}$ & $\begin{array}{l}\text { AP do FM do sexo masculino }=38,17 \mathrm{~mm} \pm 2,70 \\
\text { AP do FM do sexo feminino }=36,66 \mathrm{~mm} \pm 2,26 \\
\text { ML do FM do sexo masculino }=33,05 \mathrm{~mm} \pm 2,61 ; \\
\text { ML do FM do sexo feminino }=31,34 \mathrm{~mm} \pm 2,19 \\
\text { FM = forma redonda, em ambos os sexos. }\end{array}$ \\
\hline
\end{tabular}


Em outro aspecto, trabalhos desenvolvidos com a população indiana, mostram que estudos anatômicos ajudam no desenvolvimento de reconstruções faciais e podem proporcionar métodos atuais para determinação do sexo a partir de ossos do crânio (Biwas et al., 2015), ou seja contribuindo diretamente na anatomia forense. Nossos resultados também colaboram da mesma forma, podemos destacar que a distâncias ML dos CO do lado esquerdo no grupo feminino é maior que no masculino.

Um estudo prévio realizado no Brasil, contou com a investigação de 77 crânios e obteve as médias das mensurações muito próximas às encontradas no presente estudo, com medidas do diâmetro AP maiores que a ML, sendo o diâmetro anteroposterior médio de 34,23 $\mathrm{mm} \pm 2,54$, e o diâmetro transverso médio de 28,62 $\mathrm{mm} \pm 2,83$ (Pires et al., 2016). A forma mais identificada em ambos os trabalhos foi a oval, detectada em mais de $50 \%$ das amostras nessas pesquisas. Isso demostra uma hegemonia desse formato e nos mostra que realizar esse tipo de estudo em várias regiões do mesmo país auxilia muito o entendimento da anatomia de crânios brasileiros. Outro trabalho por exemplo, realizado por Lopez-capp et al., 2018, vai de encontro com as pesquisas até então discutidas, pois as médias ML encontradas são superiores às médias AP, dando um aspecto mais largo ao FM. Independente do resultado, todos corroboram cientificamente com o ofício de anatomistas, médicos forenses, antropólogos e de cirurgiões.

Da mesma forma, estudo realizado na Turquia, que examinou 150 crânios, a morfologia prevalente do FM foi a forma redonda, presente em 58\% das amostras (Cirpan et al., 2016). Tal achado também foi verificado em um estudo executado na Suíça, com 250 tomografias computadorizadas, no qual a configuração mais recorrente do FM, do mesmo modo, foi a forma redonda, detectado em 26\% das amostras (Edwards et al., 2013). Diferentemente das anteriores, um trabalho desenvolvido na Jordânia promoveu a investigação de 247 tomografias computadorizadas e deparou-se com a forma irregular do FM sendo hegemônica em ambos os sexos, sendo observada em 36\% das observações (Samara et al., 2017).

Outro fato que chama a atenção foi a metodologia aplicada no presente estudo, o paquímetro é um dos instrumentos mais utilizados nos estudos com abordagem morfométrica (Cirpan et al., 2016; Lopez-Capp et al., 2018; Pires et al., 2016), e mesmo quando estudos comparam medidas em crânio seco com exames de imagem (tomografia computadorizada), as médias pouco se alteram (Luzzi et al., 2019). Dessa forma, podemos destacar que nossa metodologia, apesar de ser menos complexa, apresenta eficácia.

A mensuração dos diâmetros do FM, executada no presente estudo e nos demias citados, corroboram com conhecimento científico e com aqueles seres humanos que são acometidos por afecções que comprometem o FM (Luzzi et al., 2019). Além disso, clínicos e cirurgiões podem, através de exames de imagem, identificar casos de pacientes que aperesentam um certo grau de variação anatômica, podendo ser até signficativo, além das médias elencadas na literatura, e então promover uma avaliação mais minunciosa de determinado indivíduo.

A investigação morfométrica da região basilar e condilar do osso occipital, área com alta taxa de variabilidade dimensional, é capaz de conceder dados e correlações que interferem na escolha da abordagem cirúrgica de lesões intradurais envolvendo o FM (Luzzi et al., 2019). Por se tratar de um sítio anatômico difícil, rotas cirúrgicas mais fáceis e seguras são extremamente importantes, dessa forma o estudo dos côndilos occipitais (CO) se faz de grande valia à medicina.

$\mathrm{Na}$ esfera dos $\mathrm{CO}$, nossos resultados são similaridades a outros estudos em outros países, uma vez que não encontramos na literatura estudos brasileiros para comparar com nossos resultados (Quadro 2). Em uma pesquisa realizada na Etiópia com 54 crânios secos, obteve-se médias AP para os $\mathrm{CO}$ direito e esquerdo maiores do que as detectadas no presente estudo. Porém, ao comparar as médias do diâmetro ML de ambas as pesquisas, essas são similares (Degno et al., 2019). Da mesma forma, estudos com 143 crânios de indivíduos gregos, verificou-se, da mesma forma, que as médias do diâmetro AP se apresentaram maiores, quando confrontadas com a pesquisa em vigência, porém as médias do diâmetro ML são nitidamente semelhantes (Natsis et al., 2013). Tal correlação também é vista quando se contrapõe o presente trabalho com uma pesquisa realizada com 135 crânios recuperados em Londres (Gapert, 2013). 
Quadro 2: Dados dos CO do atual estudo e dos demais estudos citados no trabalho.

\begin{tabular}{|c|c|c|c|c|}
\hline AUTOR & NACIONALIDADE & $\begin{array}{c}\text { ANO DE } \\
\text { PUBLICAÇÃO }\end{array}$ & METODOLOGIA & RESULTADOS \\
\hline $\begin{array}{l}\text { Presente } \\
\text { estudo }\end{array}$ & Brasil & 2020 & $\begin{array}{l}\text { Paquímetro Análises } \\
\text { estatísticas: Teste Qui- } \\
\text { quadrado, Teste T. }\end{array}$ & $\begin{array}{l}\text { AP do CO direito do sexo masculino }=19,81 \mathrm{~mm} ; \mathrm{AP} \\
\text { do CO esquerdo do sexo masculino }=20,43 \mathrm{~mm} \text {; ML } \\
\text { do CO direito do sexo masculino }=12,79 \mathrm{~mm} \text {; ML do } \\
\text { CO esquerdo do sexo masculino }=12,64 \mathrm{~mm} \text {; AP do } \\
\text { CO direito do sexo feminino }=21,00 \mathrm{~mm} ; \mathrm{AP} \text { do CO } \\
\text { esquerdo do sexo feminino }=21,19 \mathrm{~mm} ; \mathrm{ML} \text { do CO } \\
\text { direito do sexo feminino }=12,67 \mathrm{~mm} ; \mathrm{ML} \text { do CO } \\
\text { esquerdo do sexo feminino }=13,16 \mathrm{~mm} .\end{array}$ \\
\hline $\begin{array}{ll}\text { Natsis, } & K . \\
\text { et al }\end{array}$ & Grécia & 2013 & $\begin{array}{l}\text { Paquímetro digital } \\
\text { Análises estatísticas: } \\
\text { Teste Mann-Whitney U, } \\
\text { Teste Qui- quadrado. }\end{array}$ & $\begin{array}{l}\text { AP do CO direito }=25,60 \mathrm{~mm} \pm 2,91 ; \mathrm{ML} \text { do } \mathrm{CO} \\
\text { direito }=13,09 \mathrm{~mm} \pm 1,99 ; \mathrm{AP} \text { do } \mathrm{CO} \text { esquerdo }= \\
25,60 \mathrm{~mm} \pm 2,70 ; \mathrm{ML} \text { do } \mathrm{CO} \text { esquerdo }=13,01 \mathrm{~mm} \pm \\
1,98\end{array}$ \\
\hline $\begin{array}{l}\text { Degno, } \\
\text { Sisay et al. }\end{array}$ & Etiópia & 2018 & $\begin{array}{l}\text { Paquímetro; Análises } \\
\text { estatísticas: Teste Qui- } \\
\text { quadrado. }\end{array}$ & $\begin{array}{l}\text { AP do CO direito }=25,69 \mathrm{~mm} \pm 3,447 ; \text { AP do } \mathrm{CO} \\
\text { esquerdo }=26,96 \mathrm{~mm} \pm 3,928 ; \mathrm{ML} \text { do } \mathrm{CO} \text { direito }= \\
12,76 \mathrm{~mm} \pm 1,893 ; \mathrm{ML} \text { do } \mathrm{CO} \text { esquerdo }=13,04 \mathrm{~mm} \pm \\
2,046 .\end{array}$ \\
\hline $\begin{array}{l}\text { Gapert, } \\
\text { Black \& } \\
\text { Last. }\end{array}$ & Londres & 2013 & $\begin{array}{l}\text { Paquímetro; Análises } \\
\text { estatística: } \quad \text { Teste } \\
\text { Kolmogorov-Smirnov, } \\
\text { Teste Levene's e Box's } \\
\text { M. }\end{array}$ & $\begin{array}{l}\text { AP do CO direito no sexo masculino }=24,87 \mathrm{~mm} \pm \\
2,55 ; \mathrm{AP} \text { do CO direito no sexo feminino }=23,31 \mathrm{~mm} \\
\pm 2,33 \text { AP do CO esquerdo no sexo masculino }= \\
25,09 \mathrm{~mm} \pm 2,57 ; \text { AP do CO esquerdo no sexo } \\
\text { feminino }=23,89 \mathrm{~mm} \pm 2,45 ; \mathrm{ML} \text { do CO direito no } \\
\text { sexo masculino }=12,05 \mathrm{~mm} \pm 1,44 ; \mathrm{ML} \text { do CO direito } \\
\text { no sexo feminino }=11,50 \mathrm{~mm} \pm 1,21 ; \mathrm{ML} \text { do CO } \\
\text { esquerdo no sexo masculino }=12,13 \mathrm{~mm} \pm 1,73 ; \mathrm{ML} \\
\text { do CO esquerdo no sexo feminino }=11,61 \mathrm{~mm} \pm 1,16\end{array}$ \\
\hline
\end{tabular}

Fonte: Autores.

\section{Conclusão}

Ao final, o presente estudo constatou que os diâmetros do FM de crânios secos do Mato Grosso do Sul não apresentam diferença entre crânios masculinos e femininos, não sendo o FM uma área aconselhada para averiguação de gênero. Ao mesmo tempo foi constatado 7 morfologias distintas para o FM, sendo a forma oval a predominante. Para os CO, destacamos a média da medida ML do lado esquerdo no grupo feminino ser maior que no grupo masculino.

Nota-se que as médias das mensurações obtidas na atual pesquisa são concordantes com diversos outros estudos encontrados na literatura. Pesquisas executadas em solo africano, europeu e americano, solidificam os resultados elencados no trabalho em vigência, tantos nos parâmetros do FM quanto nos dos CO.

Apesar de estudos sobre a anatomia do FM serem frequentes, reforçamos que novas técnicas de medição e diferentes métodos de estatísticas devem ser aplicados em demais estudos para potencializar o conhecimento anatômico, antropológico e forense.

\section{Agradecimentos}

Agradecemos à Fundação Universidade Federal de Mato Grosso do Sul, campus de Três Lagoas - MS, a Pró-Reitoria de Pesquisa e Pós Graduação (PROPP) pelo Programa Institucional de Iniciação Científica Voluntária (PIVIC - edital 095/2018). 


\section{Referências}

Biswas, S., Chowdhuri, S., Das A \& Mukhopadhyay P. P. (2015). Observations on Symmetry and Sexual Dimorphism from Morphometrics of Foramen Magnum and Orbits In Adult Bengali Population. Journal Of Indian Academy Of Forensic Medicine, [s.1.], v. 37, n. 4, p.346-351, 2015. Diva Enterprises Private Limited.

Degno, S., Abrha, M, Asmare, Y. \& Muche, A. (2019) Anatomical Variation in Morphometry and Morphology of the Foramen Magnum and Occipital Condyle in Dried Adult Skulls. Journal Of Craniofacial Surgery, [S.L.], v. 30, n. 1, p. 256-259, 2019. Ovid Technologies (Wolters Kluwer Health).

Edwards, K. Viner, M. D. Schweitzer, W. \& Thali, M. J. (2013) Sex determination from the foramen magnum. Journal Of Forensic Radiology And Imaging, [S.L.], v. 1, n. 4, p. 186-192, out. 2013. Elsevier BV.

Emel, Ai., Ahmet D., A Hakan O., Engin, K., Nail, Can O., Kutluay, U., Erinc A \& Mustafa, K. B. (2010). Anatomical variations of the foramen magnum, occipital condyle and jugular tubercle. Turkish Neurosurgery, [S.L.], p. 181-190, 2010. Turkish Neurosurgical Society.

Figueiredo, E. L. de; Silva, C. P. da; Silva, M. E. L. da.; Souza neto, H. F. de; Souza, H. L. F. de; Mello, H. J. M. e; Leão J. C. \& Carvalho, A. de A. T. (2021) Malformação de Chiari e a sua correlação com transtornos orofaciais. Research, Society and Development, [S. l.], v. 10, n. 5, p. e41010515151, 2021. DOI: 10.33448/rsd-v10i5.15151. https://rsdjournal.org/index.php/rsd/article/view/15151.

Fornarino, S., Rossi, P. D., Severino, M., Pistorio, A., Allegri, A. E. M., Martelli, S., Lamba, D. L., \& Lanteri, P. (2016) Early impairment of somatosensory evoked potentials in very young children with achondroplasia with foramen magnum stenosis. Developmental Medicine \& Child Neurology, [s.1.], v. 59, n. 2, p.192-198, 16 set. 2016. Wiley.

Gerard, J. T. Princípios de Anatomia Humana. 12. ed. Rio de Janeiro: Guanabara Koogan Ltda, 2013. Tradução de: Principles of Human Anatomy, Twelfth Edition.

Gray, H. Anatomia: A base anatômica da prática clínica. 40. ed. Rio de Janeiro: Elsevier Editora Ltda, 2011. Do original: Gray's Anatomy.

Leon-Ariza, D. S., Campero, A., Chaparro, R. J. R., Prada, D. G., Grau, G. V. \& Albert L Rhoton Jr, A. L. (2017) Key Aspects in Foramen Magnum Meningiomas: From Old Neuroanatomical Conceptions to Current Far Lateral Neurosurgical Intervention. World Neurosurgery, [s.1.], v. 106, p.477-483, out. 2017. Elsevier BV.

Lima godas, A. G De; Caldeira, J. V. C; Almeida Carvalho, G. B. De., Mateus, G. B. L., Tonelli, L. B., Silva, K. R. T da \& Silva, A. V. Da. (2020). Avaliação da incidência do forame de Vesálius (forame emissário esfenoidal) em crânios secos provenientes da região centro-oeste do Brasil. Brazilian Journal of Health Review, 3(4), 8475-8487.

Lopez-Capp, T. T., Rynn, C., Wilkinson, C., Paiva, L. A. S. De., Michel-Crosato, E., Maria Gabriela Haye Biazevic, M. G. H. (2018). Sexing the Cranium from the Foramen Magnum Using Discriminant Analysis in a Brazilian Sample. Brazilian Dental Journal, [S.L.], v. 29, n. 6, p. 592-598, dez. 2018. FapUNIFESP (SciELO).

Luzzi, S., Maestro, M. Del, Elia, A., Vincitorio, F., Perna, G. Di, Zenga, F., Garbossa, D., Elbabaa, S. K., \& Renato Galzio. (2019). Morphometric and radiomorphometric study of the correlation between the foramen magnum region and the anterior and posterolateral approaches to ventral intradural lesions. Turkish Neurosurgery, [S.L.], p. 875-886, 2019. Turkish Neurosurgical Society.

Natsis, K., Piagkou, M., Skotsimara, G., Piagkos, G. \& Skandalakis, P. (2013). A morphometric anatomical and comparative study of the foramen magnum region in a Greek population. Surgical And Radiologic Anatomy, [s.1.], v. 35, n. 10, p.925-934, 26 abr. 2013. Springer Science and Business Media LLC.

Osunwoke, E.A., Oladipo, G.S., Gwunireama, I.U., \& Ngaokere, J.O. (2016). Morphometric analysis of the foramen magnum and jugular foramen in adult skulls in southern Nigerian population. American Journal Of Scientific And Industrial Research, [s.1.], v. 3, n. 6, p.446-448, dez. 2012.

Pires, L. A. S., Teixeira, A. R., Leite, T. F. O., Babinski, M. A., \& Chagas, C. A. A. (2016). Morphometric aspects of the foramen magnum and the orbit in Brazilian dry skulls. International Journal Of Medical Research \& Health Sciences. Rio de Janeiro, v. 5, n. 4, p. 34-42. jan. 2016.

René, G., Sue, B; \& Jason, L. Test of age-related variation in the craniometry of the adult human foramen magnum region: implications for sex determination methods. Forensic Science, Medicine, And Pathology, [S.L.], v. 9, n. 4, p. 478-488, 14 abr. 2013. Springer Science and Business Media LLC.

Rijken, B. F. M., Lequin, M, H., Veelen, M. C. V., Johan de Rooi J. De., \& Mathijssen, I. M. J. (2015). The formation of the foramen magnum and its role in developing ventriculomegaly and Chiari I malformation in children with craniosynostosis syndromes. Journal Of Cranio-Maxillofacial Surgery, [S.L.], v. 43, n. 7, p.1042-1048, set. 2015. Elsevier BV.

Samara, O. A., Justin Z. Amarin, J. Z., Badran, D. H., Al-Khayat, O. W., Suradi, H. H., AmShatarat, A. T., \& Hadidi, M. T. (2017). Morphometric Analysis of the Foramen Magnum. International Journal Of Morphology, [S.L.], v. 35, n. 4, p. 1270-1275, dez. 2017. SciELO Agencia Nacional de Investigacion y Desarrollo (ANID).

Sibel, C., Goksin, N. Y., Nuket, G., M., Funda, A. \& Abdurrahman, O. M. (2016). Morphological and Morphometric Analysis of Foramen Magnum. Journal Of Craniofacial Surgery, [S.L.], v. 27, n. 6, p. 1576-1578, set. 2016. Ovid Technologies (Wolters Kluwer Health).

Vanrell, J. P. (2009). Odontologia Legal \& Antropologia Forense. 2 ed. Guanabara Koogan, Rio de Janeiro, Brasil, $273,2009$. 